\title{
ANÁLISE DA VARIAÇÃO TEMPORAL DA ESTRUTURA DE UMA FLORESTA ALTIMONTANA COM HISTÓRICO DE FOGO
}

\author{
Lidiany Camila da Silva Carvalho' ${ }^{1}$ Eduardo van den Berg ${ }^{2}$, Fernando Bonillo Fernandes ${ }^{3}$
}

(recebido: 1 de junho de 2010; aceito: 22 de dezembro de 2011)

\begin{abstract}
RESUMO: Neste estudo, objetivou-se a avaliação da dinâmica estrutural de uma comunidade arbórea, com históricos de incêndios, em uma floresta estacional semidecidual altimontana, entre os anos de 2002 e 2008, na Serra da Mantiqueira. Foram calculadas taxas de dinâmica em função do número de indivíduos sobreviventes, recrutas e mortos e, taxas de dinâmica relativas ao ganho e perda de área basal. Foi verificado se existem diferenças espaciais entre as taxas ao longo de gradiente vegetacional paralelo à elevação do terreno e se há correlações entre as taxas de dinâmicas com as variáveis bióticas (número inicial de indivíduos, área basal inicial) e abiótica (cota altimétrica). Foram encontradas taxas de recrutamento superiores às taxas de mortalidade, havendo também maiores taxas de ganho em relação à perda em área basal. Esse resultado indica que a floresta se encontra em expansão, com ganho em números de indivíduos e em área basal. Quando a comunidade foi analisada em setores (base, meio e topo da encosta), verificou-se que não houve diferenças entre as taxas de dinâmicas para os setores. De maneira geral, obtiveram-se poucas correlações significativas entre as variáveis bióticas e abióticas e as taxas de dinâmicas. O ganho em número de indivíduos e área basal, a não diferenciação das taxas de dinâmica entre os setores e as baixas correlações entre as variáveis com dinâmica da estrutura da floresta são evidências que eventos históricos, como incêndio ocorrido na década de 90, podem estar afetando diretamente as taxas de dinâmica da floresta como um todo.
\end{abstract}

Palavras-chave: Gradiente vegetacional, dinâmica estrutural, incêndio.

\section{ANALYSIS OF THE TEMPORAL VARIATION OF THE STRUCTURE OF A MONTANE FOREST WITH HISTORICAL OF FIRE}

\begin{abstract}
The aim of this study was to evaluate the structural dynamic rates of an shrubs-tree component of a seasonal semideciduous upper montane forest, in Mantiqueira Mountain between 2002 and 2008. We calculated the rates of dynamic according to the number of surviving, dead individuals and recruits, as well as the rates of dynamic for gain and loss of basal area. We verified the spatial differences among the rates along the vegetation gradient parallel to ground elevation. We also studied the correlations between the rates and biotic (initial numbers of trees and initial basal area) and abiotic parameters (altimetric quota). We verified that recruitment was higher than mortality, and the gain of basal area was higher than the loses. This result suggests that the forest is expanding, with gain in number of individuals and in basal area. Normally, this result characterizes forests in recuperation after some disturbance. The community sectors (basis, middle and top of hillside) didn't show any differences in terms of dynamic rates. In general, there were few significant correlations between biotic and abiotic parameters and the dynamic rates. The increase of density and basal area, the similarity of dynamic rates among the sectors and the low correlation between parameters and the dynamic of forest's structure point out that the forest burning occurred in 90 's could be, nowadays, interfering directly in dynamic rates of forest.
\end{abstract}

Key words: Vegetation gradient, structural dynamic, burning.

\section{INTRODUÇÃO}

As florestas tropicais altimontanas normalmente são distintas em termos de composição florística e estrutural em relação às florestas de baixas altitudes (AIBA; KITAYAMA, 1999; HOMEIER et al., 2008; LEUSCHNER et al., 2007; LIEBERMAN et al., 1996). Como ocupam encostas declivosas essas florestas apresentam mudança rápida na composição de espécies e estrutura ao longo do gradiente altitudinal (WHITMORE, 1990).
Alguns estudos relacionam esse gradiente a heterogeneidade ambiental ligada às condições hídricas e fatores edáficos associados ao relevo (GARTLAN et al., 1986; HOMEIER et al., 2004, 2008).

Existem poucos levantamentos florístico-estruturais de vegetação florestal altimontana na região sudeste do Brasil, destacando-se os trabalhos de Carvalho et al. (2005), França e Stehmann (2004) e Pereira et al. (2006). Esses trabalhos enfocam, sobretudo, a descrição florística e fitossociológica da comunidade florestal. Portanto, são

\footnotetext{
${ }^{1}$ Engenheira Florestal, Mestre em Engenharia Florestal - Laboratório de Ciências Ambientais - Universidade Estadual do Norte Fluminense/UENF Rua Coronel Pinto, 341 -69301-150 - Boa Vista, RR - lidycamila@yahoo.com.br

${ }^{2}$ Engenheiro Agrônomo, Professor Doutor em Biologia Vegetal - Departamento de Biologia - Universidade Federal de Lavras/UFLA - Cx. P. 3037 37200-000 - Lavras, MG - eduardo.lavras@gmail.com

${ }^{3}$ Biólogo, Doutor em Engenharia Florestal - Rua Francisco Sales, 171, Centro - 37.550-000 - Pouso Alegre, MG - bonillofernandes@uol.com.br
}

Cerne, Lavras, v. 18, n. 2, p. 223-230, abr./jun. 2012 
escassas as informações sobre mudanças estruturais, ao longo do tempo, desses tipos de vegetação e pouco se sabe sobre quais fatores podem estar associados ao processo de dinâmica dessas florestas. O que se tem conhecimento é que o processo de dinâmica pode ser afetado e direcionado por distúrbios (JENTSCH et al., 2002).

Neste estudo, objetivou-se a avaliação da dinâmica de um fragmento de Floresta Estacional Semidecidual Altimonta, entre os anos 2002 e 2008. Buscou-se verificar se há variações nas taxas de dinâmica em relação ao gradiente vegetacional existente e averiguar se essas taxas estão desequilibradas, apontando ganho em número de indivíduos e em área basal, em decorrência do processo de regeneração em que a floresta se encontra em função de um incêndio ocorrido na década de 1990. As hipóteses testadas foram: 1) As taxas de dinâmica variam da base ao topo da encosta, refletindo as mudanças florísticas, topográficas e edáficas observadas na área. 2) $O$ incremento em área basal diminui com o aumento da altitude, uma vez que, em elevadas altitudes, as plantas tendem a ter uma menor produtividade líquida e, consequentemente, um menor crescimento. 3) Nas parcelas mais densas ou com maior área basal há maior mortalidade, maior perda de área basal, menor recrutamento e menor ganho em área basal, em razão da maior competição experimentada. 4) A floresta se encontra em um processo de construção após um incêndio ocorrido na década de 1990 e apresenta taxas de mudança positivas para números de indivíduos e área basal.

\section{MATERIAL E MÉTODOS}

\subsection{Caracterização da área de estudo}

O estudo foi conduzido no Município de Poços de Caldas, Minas Gerais, Brasil, em um fragmento localmente conhecido como Mata da Colina, localizado dentro dos limites $46^{\circ} 31^{\prime}$ '03' $\mathrm{W}, 46^{\circ} 31^{\prime} 27^{\prime}$ 'W, $21^{\circ} 46^{\prime} 36^{\prime \prime} \mathrm{S}$ e 21 ${ }^{\circ} 46^{\prime} 51^{\prime \prime}$ 'S. Segundo Fernandes (2003), a área não tem sofrido impactos antrópicos sistemáticos. Porém, existem relatos de um incêndio florestal ocorrido na década de 90 que, provavelmente, atingiu toda a área estudada, embora as informações sejam escassas sobre a sua intensidade e abrangência. $\mathrm{O}$ solo do fragmento foi classificado como Cambissolo Húmico Distrófico Léptico de acordo com a Empresa Brasileira de Pesquisa Agropecuária - Embrapa (2000). O clima predominante na região com base na classificação de Koppen é Cfb (subtropical úmido) (INSTITUTO DE DESENVOLVIMENTO INDUSTRIAL - INDI, 1977), e o índice pluviométrico anual é de $1.695 \mathrm{~mm}$.
A temperatura média anual é de $17,6^{\circ} \mathrm{C}$; com umidade relativa anual média de 78,2\% (GISLER, 1995). A altitude no fragmento varia de $1400 \mathrm{~m}$ a $1643 \mathrm{~m}$, enquadrando-se no sistema de classificação proposto por Oliveira Filho e Fontes (2000), nos domínios fitoecológicos das florestas estacionais semideciduais altimontanas.

\subsection{Inventário da comunidade arbórea}

O levantamento estrutural foi realizado entre junho e outubro de 2002 e os resultados se encontram em Fernandes (2003). O desenho amostral (Figura 1) foi constituído por 28 parcelas permanentes de $400 \mathrm{~m}^{2}$ distribuídas sistematicamente ao longo de quatro transectos. Dentro da área delimitada pelas parcelas, todos os indivíduos que apresentaram circunferência à altura do peito (circunferência à altura de $1,30 \mathrm{~m}$ ) ou a soma quadrática dos CAP's dos indivíduos ramificados igual ou superior a $15,7 \mathrm{~cm}$ foram marcados e tiveram seu CAP medido. A identificação botânica foi realizada por especialista no próprio local, ou por meio de coleta do material botânico e consulta posterior a herbários, especialista ou literatura pertinente.

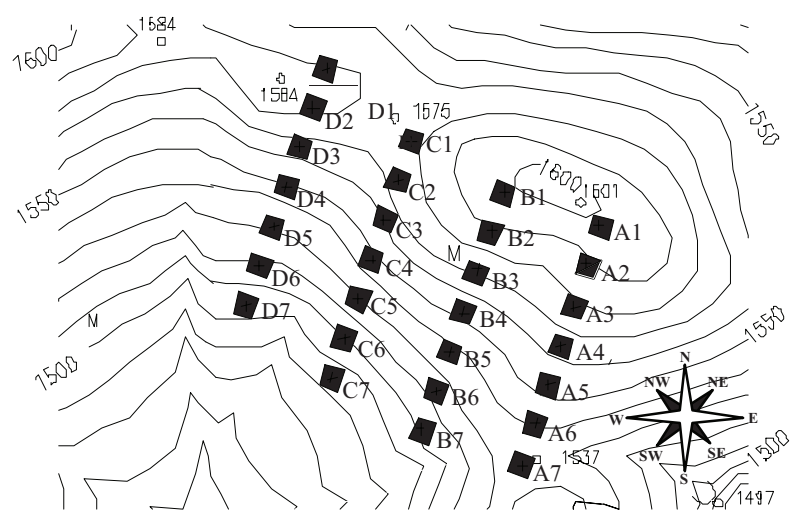

Figura 1 - Disposição dos transectos e respectivas parcelas; as linhas contínuas representam as curvas de nível, no fragmento florestal da Mata da Colina, Poços de Caldas, MG.

Figure 1 - Layout of transects and their plots; the solid lines represent the curves of levels in the forestry fragment of Mata da Colina, Poços de Caldas, $M G$.

Em julho de 2008, foi realizado o segundo inventário onde os indivíduos mortos foram contabilizados, os sobreviventes foram novamente medidos e os recrutas (indivíduos que alcançaram o critério mínimo de inclusão), foram marcados, medidos e identificados. O intervalo de tempo entre os pontos centrais da duração dos dois inventários foi de 6,12 anos.

Cerne, Lavras, v. 18, n. 2, p. 223-230, abr./jun. 2012 


\subsection{Análise da dinâmica da comunidade arbórea}

As taxas de dinâmica foram calculadas para a amostra total da comunidade e para extrações da amostra total definidas pelas parcelas amostrais representando a base, meio e topo do gradiente (Figura 1). Cada estrato foi composto de sete parcelas sorteadas aleatoriamente - base (A6, A7, B6, B7, C6, C7 e D6), meio (A4, B3, B4, C3, C5, D4 e D5) e topo (A1, A2, B1, C1, C2, D1 e D2). A dinâmica foi analisada de duas formas: em número (contagens) de indivíduos arbóreos (recrutas, mortos e sobreviventes) e em área basal das árvores. Com base em Sheil et al. (1995, 2000), foram calculadas as taxas anuais médias de mortalidade $\left(M=\left\{1-\left[\left(\mathrm{N}_{0}-m\right) / N_{0}\right]^{1 / t}\right\} \times 100\right)$ e recrutamento $\left(R=\left[1-(1-r / N \mathrm{t})^{1 / t}\right] \times 100\right)$ de árvores individuais e taxas anuais médias de perda $\left(P=\left\{1-\left[(A B \mathrm{t}-A B \mathrm{~m}-A B \mathrm{~d}) / A B_{0}\right]^{1 / \mathrm{t}}\right\}\right.$ $\times 100)$ e ganho $\left(G=\left\{1-[1-(A B \mathrm{r}+A B \mathrm{~g}) / A B \mathrm{t}]^{1 / t}\right\} \times 100\right)$ de área basal das árvores. Sendo t o tempo decorrido entre os inventários (6,12 anos), $N_{0}$ e $\mathrm{Nt}$, respectivamente, as contagens inicial e final de árvores individuais; $m$ e $r$ são, respectivamente, o número de árvores mortas e recrutas; $A B \mathrm{t}$ e $A B \mathrm{t}$ são, respectivamente, as áreas basais inicial e final das árvores; $A B \mathrm{~m}$ é a área basal das árvores mortas, $A B r$ é a área basal dos recrutas; e $A B \mathrm{~d}$ e $A B g$ são, respectivamente, o decremento e o incremento em área basal das árvores sobreviventes. Foram obtidas também as taxas de rotatividade em número de árvores $(T \mathrm{~N}=(M+R) / 2) \mathrm{e}$ área basal $(T A B=(P+G) / 2)$ (OLIVEIRA FILHO et al., 1997). De acordo com Korning e Balslev (1994), também foram ainda obtidas as taxas de mudança líquida no período, tanto para número de árvores $\left(C h \mathrm{~N}=\left[\left(N_{\mathrm{t}} / N_{0}\right)^{1 / \mathrm{t}}-1\right] \times 100\right)$ como área basal $\left(C h \mathrm{AB}=\left[\left(A B_{\mathrm{t}} / A B_{0}\right)^{1 / \mathrm{t}}-1\right] \times 100\right)$.

As taxas de dinâmica para os três setores (base, meio e topo) foram comparadas pela ANOVA não paramétrica de Kruskal-Wallis, para um $P \leq 0,05$. O teste Mann-Whitney foi utilizado para verificação das diferenças entre as taxas de mortalidade e recrutamento, perda e ganho em área basal dentro de cada setor ou para a comunidade como um todo (SOKAL; ROHLF, 1995). A influência das variáveis número inicial de indivíduos, área basal inicial e a cota sobre a taxa de dinâmica da comunidade (taxa de recrutamento, mortalidade, incremento área basal) foram testadas por meio de regressão linear simples.

\section{RESULTADOS}

Em 2002, foram amostrados 1337 indivíduos e, em 2008, 1444. Foram encontrados 186 indivíduos mortos e 293 recrutas. Para a área amostrada como um todo, a taxa de mortalidade foi de $2,41 \% \mathrm{ano}^{-1}$ e o recrutamento de $3,64 \%$ ano $^{-1}$, resultando nas taxas de rotatividade de $3,03 \% \mathrm{ano}^{-1}$ e na de mudança de $1,26 \% \mathrm{ano}^{-1}$. As taxas de dinâmica não diferiram entre os setores (Tabela 1). Ao analisar a dinâmica dentro de cada setor, percebe-se que as taxas de recrutamento foram superiores às taxas de mortalidade no topo $(\mathrm{P}=0,04876)$ e na base $(\mathrm{P}=0,04612)$ e para a amostra total $(\mathrm{P}=0,03763)$.

A área total amostrada apresentou uma área basal inicial (2002) de $19,05 \mathrm{~m}^{2}$ e área basal final (2008) de $22,73 \mathrm{~m}^{2}$, sendo a área basal dos indivíduos mortos $1,81 \mathrm{~m}^{2} \mathrm{e}$, recrutas, $0,95 \mathrm{~m}^{2}$. A taxa de ganho foi de $4,79 \% \mathrm{ano}^{-1}$ e a taxa de perda de $2,00 \% \mathrm{ano}^{-1}$, e as taxas de rotatividade e mudança líquida em área basal foram de $3,40 \% \mathrm{ano}^{-1}$ e $2,92 \% \mathrm{ano}^{-1}$, respectivamente. As taxas de ganho foram superiores à taxa de perda no topo $(\mathrm{P}=0,03605)$, na base da encosta $(\mathrm{P}=0,046814)$ e na amostra total $(\mathrm{P}=0,01879)$. O teste de Kruskal-Wallis não mostrou diferenças significativas para as taxas de dinâmica de área basal entre os setores (Tabela 1).

Para as regressões, foram encontrados de modo geral, baixos coeficientes de correlação (Tabela 2). A cota apresentou correlação negativa apenas com o incremento em área basal $\left(b=-0,0025, \mathrm{R}^{2}=0,21 \mathrm{e} \mathrm{P}<0,0131\right)$. Houve correlação positiva entre o número de indivíduos inicial (Ni) com o incremento em área basal dos sobreviventes $\left(\mathrm{b}=0,0032, \mathrm{R}^{2}=0,23\right.$ e $\left.\mathrm{P}<0,0097\right)$. Á área basal inicial (ABi) teve correlações negativa para a taxa de recrutamento $\left(\mathrm{b}=-2,1736, \mathrm{R}^{2}=0,15\right.$ e $\left.\mathrm{P}<0,0387\right)$ e positiva para $\mathrm{o}$ incremento em área basal $\left(b=0,216, R^{2}=0,31\right.$ e $\left.P<0,0019\right)$. A mortalidade não se mostrou correlacionada com nenhuma das variáveis testadas (Tabela 2).

\section{DISCUSSÃO}

A Mata da Colina possui um gradiente vegetacional representado por formações mais exuberantes na base e nas linhas de drenagem e por formações escleromórficas de baixa estatura, principalmente no meio da encosta (FERNANDES, 2003). Apesar desse gradiente vegetacional, os resultados encontrados demonstram que não existem diferenças nas taxas de dinâmica entre os setores. Portanto, o processo de dinâmica estrutural da floresta acontece de maneira similar ao longo de todo o gradiente. Embora a intensidade e abrangência do incêndio florestal ocorrido em meados dos anos 90 não sejam conhecidas, o impacto desse evento sobre a estrutura da floresta parece ser ainda bastante evidente. Segundo Hoffman e Moreira (2002), um incêndio pode alterar substancialmente a estrutura e dinâmica das populações dentro de uma comunidade vegetal. Esse, provavelmente, foi o fator responsável pela ausência de diferenciação das taxas de dinâmica entre os setores.

Cerne, Lavras, v. 18, n. 2, p. 223-230, abr./jun. 2012 
Tabela 1 - Dinâmica da comunidade arbórea em uma floresta tropical altimontana inventariada nos anos de 2002 e 2008 em Poços de Caldas, MG, contabilizada para a amostra total e seus três setores, base, meio e topo, e expressa em número de árvores e área basal. Diferenças entre os setores foram avaliadas pelo teste de Kruskal-Wallis $(\mathrm{P} \leq 0,05)$. Diferenças entre as taxas dentro dos setores ou para a comunidade foram avaliadas pelo Teste de Mann-Whitney $(\mathrm{P} \leq 0,05)$

Table 1 - Tree community dynamics in a tropical altimountain forest surveyed in 2002 and 2008 in Poços de Caldas, SE Brazil, given for the total sample and its three strata, base, middle and top, expressed as number of trees and tree basal area. Differences among strata were evaluated by Kruskal-Wallis test $(P \leq 0,05)$. Differences between rates inside the strata or for the community were evaluated by Mann-Whitney test $(P \leq 0,05)$.

\begin{tabular}{|c|c|c|c|c|}
\hline & \multirow{2}{*}{ Amostra total } & \multicolumn{3}{|c|}{ Setor } \\
\hline & & Topo & Meio & Base \\
\hline $\mathrm{N}$ parcelas & 28 & 7 & 7 & 7 \\
\hline Número inicial indivíduos & 1337 & 402 & 269 & 354 \\
\hline Número final indivíduos & 1444 & 432 & 289 & 386 \\
\hline Indivíduos sobreviventes & 1151 & 346 & 228 & 314 \\
\hline Indivíduos mortos & 186 & 56 & 41 & 40 \\
\hline Indivíduos recrutas & 293 & 86 & 61 & 72 \\
\hline Taxa de mortalidade $\left(\%\right.$. ano $\left.^{-1}\right)$ & 2,41 & 2,42 & 2,66 & 1,94 \\
\hline Kruskal-Wallis (P) (mortalidade) & & & 0,5853 & \\
\hline Taxa de recrutamento $\left(\%\right.$. ano $\left.^{-1}\right)$ & 3,64 & 3,56 & 3,8 & 3,32 \\
\hline Kruskal-Wallis (P) (recrutamento) & & & 0,8913 & \\
\hline Mann-Whitney (P) (mortalidade x recrutamento) & 0,0376 & 0,0487 & 0,8279 & 0,0461 \\
\hline Taxa de rotatatividade $\left(\%\right.$. ano $\left.^{-1}\right)$ & 3,03 & 2,99 & 3,23 & 2,63 \\
\hline Kruskal-Wallis (P) (rotatividade) & & & 0,447 & \\
\hline Taxa de mudança $\left(\% \cdot\right.$ ano $\left.^{-1}\right)$ & 1,26 & 1,18 & 1,18 & 1,42 \\
\hline Kruskal-Wallis (P) (mudança) & & & 0,9319 & \\
\hline Área basal inicial $\left(\mathrm{m}^{2}\right)$ & 19,0473 & 4,0359 & 4,0278 & 7,7578 \\
\hline Final $\left(\mathrm{m}^{2}\right)$ & 22,7299 & 4,8131 & 4,2669 & 9,4894 \\
\hline Mortos $\left(\mathrm{m}^{2}\right)$ & 1,8118 & 0,3601 & 0,4967 & 0,5121 \\
\hline Decremento dos sobreviventes $\left(\mathrm{m}^{2}\right)$ & 0,3969 & 0,0445 & 0,1618 & 0,1292 \\
\hline Incrementos dos sobreviventes $\left(\mathrm{m}^{2}\right)$ & 4,8319 & 0,8115 & 0,6854 & 2,1027 \\
\hline Indivíduos recrutas $\left(\mathrm{m}^{2}\right)$ & 0,9513 & 0,3704 & 0,2000 & 0,2657 \\
\hline Taxa de perda $\left(\%\right.$. ano $\left.^{-1}\right)$ & 2,00 & 1,71 & 2,87 & 1,40 \\
\hline Kruskal-Wallis (P) (Taxa de perda) & & & 0,3699 & \\
\hline Taxa de ganho $\left(\%\right.$. ano $\left.^{-1}\right)$ & 4,79 & 4,50 & 3,77 & 4,58 \\
\hline Kruskal-Wallis (P) (Taxa de ganho) & & & 0,5071 & \\
\hline Mann-Whitney (P) (Tx. ganho x Tx. perda) & 0,01879 & 0,0365 & 0,2098 & 0,0468 \\
\hline Taxa de rotatividade $\left(\%\right.$. ano $\left.^{-1}\right)$ & 3,39 & 3,10 & 3,32 & 2,99 \\
\hline Kruskal-Wallis $(\mathrm{P})$ (Taxa de rotatividade) & & & 0,7915 & \\
\hline Taxa de mudança $\left(\%\right.$. ano $\left.^{-1}\right)$ & 2,92 & 2,92 & 0,95 & 3,34 \\
\hline Kruskal-Wallis (P) (Taxa de mudança) & & & 0,3237 & \\
\hline
\end{tabular}

Cerne, Lavras, v. 18, n. 2, p. 223-230, abr./jun. 2012 
Tabela 2 - Regressões lineares para amostra total do componente arbustivo-arbóreo da floresta altimontana, em Poços de Caldas, MG, Brasil, relacionando o número de indivíduos inicial, a área basal inicial e a cota altimétrica com as taxas de dinâmica e incremento em área basal para o período de 2002-2008 ( Ni = número inicial de indivíduos; $\mathrm{ABi}$ = área basal inicial, Cota = cota altimétrica; $\mathrm{x}$ = variável independente; $\mathrm{y}=$ variável dependente; Equação = equação de regressão linear; $\mathrm{R}^{2}=$ coeficiente de determinação; $\mathrm{F}=\mathrm{o}$ valor do teste $\mathrm{F} ; \mathrm{P}=$ nível de significância); ${ }^{*} P \leq 0,05$.

Table 2 - Linear regressions for the total sample for the shrub-tree component of upper montaine forest in Poços de Caldas, Minas Gerais State, Brazil, relating the initial number of individuals, the basal area and altimetric quota to the dynamic rates and increment in basal area for the period 2002-2008 (Ni = initial number of individuals; ABi = initial basal area; Cota = altimetric quota; $x=$ independent variable; $y=$ dependent variable; Equation $=$ linear regression equation; $R^{2}=$ determination coefficient $; F=F$ test value; $P=$ significance level); * $P \leq 0,05$.

\begin{tabular}{lccccc}
\hline $\mathrm{X}$ & $\mathrm{Y}$ & Equação & $\mathrm{R}^{2}$ & $\mathrm{~F}$ & $\mathrm{P}$ \\
\hline $\mathrm{Ni}$ & $\mathrm{Tx}$, Mortalidade & $\mathrm{y}=4,9677-0,0426^{*} \mathrm{x}$ & 0,0856 & 2,43 & 0,1309 \\
$\mathrm{Ni}$ & Tx, Recrutamento & $\mathrm{y}=4,5851-0,0170^{*} \mathrm{x}$ & 0,032 & 0,86 & 0,3627 \\
$\mathrm{Ni}$ & Incremento AB & $\mathrm{y}=0,0058+0,0032^{*} \mathrm{x}$ & 0,2306 & 7,94 & $0,0097 *$ \\
$\mathrm{ABi}$ & $\mathrm{Tx}$, Mortalidade & $\mathrm{y}=4,1790-1,8286^{*} \mathrm{x}$ & 0,0464 & 1,27 & 0,2707 \\
$\mathrm{ABi}$ & Tx, Recrutamento & $\mathrm{y}=5,2536-2,1736^{*} \mathrm{x}$ & 0,1543 & 4,74 & $0,0387 *$ \\
$\mathrm{ABi}$ & Incremento AB & $\mathrm{y}=0,0105+0,2160^{*} \mathrm{x}$ & 0,3138 & 11,89 & $0,0019 *$ \\
$\mathrm{Cota}$ & Tx, Mortalidade & $\mathrm{y}=-43,9958+0,0302 * \mathrm{x}$ & 0,0659 & 1,83 & 0,1874 \\
$\mathrm{Cota}$ & Tx, Recrutamento & $\mathrm{y}=-4,3004+0,0052^{*} \mathrm{x}$ & 0,0046 & 0,11 & 0,7321 \\
$\mathrm{Cota}$ & Incremento AB & $\mathrm{y}=4,0056-0,0025^{*} \mathrm{x}$ & 0,2144 & 7,09 & $0,0131 *$ \\
\hline
\end{tabular}

Após um incêndio, normalmente a floresta passa por um primeiro estágio de elevada mortalidade (SHEIL; BURSLEM, 2003), principalmente de indivíduos menores que são menos resistentes, alterando as densidades e as estruturas populacionais. Essa fase de depleção é seguida por um período de recuperação, onde ocorre o recrutamento de uma nova coorte (OGDEN et al., 1998), resultando no ganho em densidade de indivíduos, área basal e, muitas vezes, na substituição de espécies (CASTELLANI; STUBBLEBINE, 1993). Portanto, esses distúrbios podem afetar as taxas de mortalidade, recrutamento e rotatividade em número de indivíduos (SHEIL, 1995), influenciando a estrutura e a dinâmica temporal das florestas (WHITE; PICKETT, 1985).

Embora a comunidade como um todo tenha apresentado taxas positivas quanto ao ganho em indivíduos e em área basal, quando se analisam os setores individualmente, observa-se que o meio, ao contrário dos demais, apresentou taxas de mortalidade e recrutamento, ganho e perda em área basal estatisticamente iguais entre si. Tal comportamento pode ser atribuído à peculiaridades deste setor. O afloramento de bauxita concentrado em muitos pontos desse setor observado por Fernandes (2003) pode estar agindo como um impedimento físico à ocupação desse espaço por novos indivíduos. Além disso, a vegetação herbácea, com expressiva ocorrência de Pteridium aquilinum (FERNANDES, 2003) pode representar um impedimento adicional à ocupação por espécies arbóreas. $P$. aquilinum é uma colonizadora agressiva e pode estar atuando como um competidor por recursos. A grande produtividade dessa espécie e o acúmulo da serrapilheira resultante dificultam a entrada de luz para a germinação e o estabelecimento do banco de sementes (GHORBANI et al., 2003), dificultando o processo de regeneração natural.

A correlação negativa entre a variável cota e o incremento em área basal dos indivíduos sobreviventes é um padrão tipicamente observado em gradientes altitudinais. Alguns estudos têm verificado resultado similar, com um decremento na produtividade primária líquida e um crescimento mais lento das árvores com o aumento da altitude, e, consequentemente, um menor incremento em área basal (HOMEIER et al., 2004, 2008; LEUSCHNER et al., 2007). Essa relação entre a taxa de incremento e a altitude em áreas declivosas pode estar associada à variação na concentração e disponibilidade de nutrientes que podem existir, em razão da lixiviação de nutrientes ao longo da encosta e ao acúmulo desses sedimentos nas depleções ou nas áreas de menor altitude (SOETHE et al., 2008), além das variações microclimáticas (HOMEIER et al., 2004).

Cerne, Lavras, v. 18, n. 2, p. 223-230, abr./jun. 2012 
A correlação negativa entre área basal inicial e o recrutamento também foi encontrada em outros trabalhos de dinâmica (FELFILI, 1995; GUIMARÃES et al., 2008). A redução do recrutamento com o aumento da área basal inicial pode refletir uma condição de competição entre os indivíduos. Normalmente, área basal elevada está associada a uma vegetação mais densa ou com indivíduos maiores, onde há uma menor disponibilidade de luz para os indivíduos mais jovens, retardando o crescimento desses indivíduos do sub-bosque e, consequentemente, diminuindo o recrutamento (LAURANCE et al., 2006; MICHALSKI et al., 2007). A relação negativa da área basal inicial com o recrutamento é bem comum e, em alguns casos, tem sido utilizada como parâmetro na modelagem do recrutamento em florestas nativas como os modelos propostos por Kohyama (1992) e Spathelf e Durlo (2001).

Considerando os resultados encontrados para o intervalo de tempo avaliado, o ganho em número de indivíduos e área basal indica que a floresta esteja em meio a um processo de construção, recuperando-se de algum distúrbio provavelmente $\mathrm{o}$ incêndio ocorrido em meados dos anos 90. Efeitos de outras formas de distúrbios como o corte seletivo de madeira e o gado têm sido documentados e discutidos em outros trabalhos que abordam dinâmica de florestas tropicais (HIGUCHI et al., 2008; OLIVEIRA FILHO et al., 2007; WERNECK; FRANCESCHINELLI, 2004). Supondo que não haja novos distúrbios causadores de impactos significativos na vegetação arbórea, as taxas de dinâmicas, principalmente de mortalidade e recrutamento provavelmente tenderão a um equilíbrio, com uma desaceleração na taxa de ganho em área basal (OLIVEIRAFILHO et al., 1997). Isso poderá ser verificado em inventários futuros, onde, possivelmente, a floresta entrará em um processo de construção tardia, caracterizada pelo autodesbaste, no qual a densidade de indivíduos arbóreos diminuiria, principalmente nas classes de menor diâmetro, e a área basal aumentaria ou estabilizaria (GENTRY; TERBORGH, 1992; OLIVEIRA FILHO et al., 1997, 2007; WERNECK; FRANCESCHINELLI, 2004). Outra possibilidade é que a comunidade apresente um padrão de constante desequilíbrio, pelas frequentes perturbações naturais ou antrópicas, como por exemplo, incêndios recorrentes, que podem causar alterações permanentes na estrutura de florestas tropicais e no ciclo de dinâmica (COCHRANE; SCHULZE, 1999). Nesse caso, a comunidade se mostraria sempre instável, com oscilações cíclicas nas taxas de mortalidade e recrutamento impedindo que a floresta atinja seu equilíbrio (SWAINE et al., 1987).

\section{CONCLUSÕES}

Quanto aos resultados obtidos, ao contrário do que se esperava na hipótese 1 , as taxas de dinâmica não variaram entre os setores. Esse resultado fortalece a discussão de que o incêndio ocorrido na década de 90 , que atingiu toda a comunidade, esteja hoje sendo um dos principais fatores no direcionamento e na homogeneização das taxas encontradas para a dinâmica estrutural em todos os setores. A hipótese 2 foi aceita diante da relação negativa entre a altitude e o incremento em área basal. A hipótese 3 foi aceita apenas para relação negativa entre a área basal inicial e o recrutamento. No entanto, tanto a densidade quanto a área basal aparentemente não afetaram a mortalidade ou a perda de área basal. O processo de dinâmica da Mata da Colina dentro do intervalo de tempo estudado se mostra mais influenciado pelo incêndio ocorrido no passado do que pelas próprias variáveis bióticas como a diferença estrutural ao longo da encosta encontrada no primeiro levantamento em 2002. Muitos dos resultados encontrados caracterizam uma floresta em expansão em número de indivíduos e biomassa e sugerem que a comunidade florestal estudada esteja em processo de reconstrução, em resposta a uma perturbação, o que confirma a hipótese 4 .

\section{REFERÊNCIAS}

AIBA, S.; KITAYAMA, K. Structure, composition and species diversity in an altitudesubstrate matrix of rain forest tree communities on Mount Kinabalu, Borneo. Plant Ecology, Dordrecht, v. 140, n. 2, p. 139-157, Feb. 1999.

CARVALHO, D. A.; OLIVEIRA FILHO, A. T.; BERG, E. van den; FONTES, M. A. L.; VILELA, E. A.; MARQUES, J. J. G. S. M.; CARVALHO, W. A. C. Variações florísticas e estruturais do componente arbóreo de uma floresta ombrófila alto-montana às margens do Rio Grande, Bocaina de Minas, MG, Brasil. Acta Botanica Brasílica, Porto Alegre, v. 19, n. 1, p. 91-109, Feb. 2005.

CASTELLANI, T. T.; STUBBLEBINE, W. H. Sucessão secundária inicial em uma mata tropical, apos perturbação por fogo. Revista Brasileira de Botânica, São Paulo, v. 16, n. 2, p. 181-203, abr. 1993.

COCHRANE, M. A.; SCHULZE, M. D. Fire as a recurrent event in tropical forests of the eastern Amazon: effects on forest structure, biomass and species composition. Biotropica, Washington, v. 31, n. 1, p. 2-16, Feb. 1999.

Cerne, Lavras, v. 18, n. 2, p. 223-230, abr./jun. 2012 
EMPRESA BRASILEIRA DE PESQUISA

AGROPECUÁRIA. Centro Nacional de Pesquisa de Solos. Sistema brasileiro de classificação de solos. Brasília, 2000. $412 \mathrm{p}$.

FELFILI, J. M. Diversity, structure and dynamics of a gallery forest in central Brazil. Vegetatio, The Hague, v. 117, n. 1, p. 1-15, Mar. 1995.

FERNANDES, F. B. Estudo de gradientes vegetacionais em uma floresta semidecídua altimontana no Planalto de Poços de Caldas, MG. 2003. 158 p. Dissertação (Mestrado em Engenharia Florestal) - Universidade Federal de Lavras, Lavras, 2003.

FRANÇA, G. S.; STEHMANN, J. R. Composição florística e estrutura do componente arbóreo de uma floresta altimontana no município de Camanducaia, Minas Gerais, Brasil. Revista Brasileira de Botânica, São Paulo, v. 27, n. 1, p. 19-30, mar. 2004.

GARTLAN, J. S.; NEWBERY, D. M.; THOMAS, D. W.; WATERMAN, P. G. The influence of topography and soil phosphorus on the vegetation of Korup Forest Reserve, Cameroun. Vegetatio, The Hague, v. 65, n. 3, p. 131-148, Sept. 1986.

GENTRY, A. H.; TERBORGH, J. Composition and dynamics of the Cosha Cashu 'mature' floodplain forest. In: GENTRY, A. H. (Ed.). Four neotropical rainforests. New Haven: Yale University, 1990. p. 542-564.

GHORBANI, J. A.; DAS, P. M.; DAS, A. B.; HUGHES, J. M.; MCALLISTER, H. A.; PALLAI, S. K.; PAKEMANR, J.; MARRS, R. H.; LE DUC, M. G. Effects of restoration trataments on the diaspore bank under dense Pteridium stands in the UK. Applied Vegetation Science, Knivsta, v. 6, n. 2, p. 189-198, June 2003.

GISLER, C. V. T. O uso da serapilheira na recomposição da cobertura vegetal em áreas mineradas de bauxita, Poços de Caldas, MG. 1995. 133 f. Dissertação (Mestrado em Ecologia) - Universidade de São Paulo, São Paulo, 1995.

GUIMARÃES, J. C. C.; BERG, E. V. D.; CASTRO, G. C.; MACHADO, E. L. M.; OLIVEIRA FILHO, A. T. Dinâmica do componente arbustivo-arbóreo de uma floresta de galeria aluvial no planalto de Poços de Caldas, MG, Brasil. Revista Brasileira de Botânica, São Paulo, v. 31, n. 4, p. 621-632, dez. 2008.
HIGUCHI, P.; OLIVEIRA FILHO, A. T.; BEBBER, D. P.; BROWN, N. D.; SILVA, A. C.; MACHADO, E. L. M. Spatio-temporal patterns of tree community dynamics in a tropical forest fragment in South-east Brazil. Plant Ecology, Dordrecht, v. 199, n. 1, p. 125-135, Nov. 2008.

HOFFMANN, W. A.; MOREIRA, A. G. The role of fire in population dynamics of woody plants. In: OLIVEIRA, P. S.; MARQUIS, R. J. (Ed.). The Cerrados of Brazil: ecology andnatural history of a neotropical savanna. New York: Columbia University, 2002. p. 159-177.

HOMEIER, J.; WALDSTRUKTUR, W.; YNAMIK, Z. Tropischer bergregenwälder in ecuador und Costa Rica. Dissertationes Botanicae, San José, v. 391, n. 1, p. 1-27, 2004.

HOMEIER, J.; WERNER, F. A.; GRADSTEIN, S. R.; BRECKLE, S. W.; RICHTER, M. Potential vegetation and floristic composition of Andean forests in South Ecuador, with a focus on the RBSF. In: BECK, E.; BENDIX, J.; KOTTKE, I.; MAKESCHIN, F.; MOSANDL, R. (Ed.). Gradients in a tropical mountain ecosystem of Ecuador. Berlin: Ecological Studies Springer, 2008. p. 87-100.

INSTITUTO DE DESENVOLVIMENTO INDUSTRIAL. Informações básicas para investidores. Belo Horizonte, 1977. 189 p.

JENTSCH, A.; BEIERKUHNLEIN, C.; WHITE, A. S. Scale, the dynamic stability of forest ecosystems, and the persistence of biodiversity. Silva Fennica, Helsinski, v. 36, n. 1, p. 393402, Feb. 2002.

KOHYAMA, T. Size-structured multi-species model of rain forest trees. Functional Ecology, Oxford, v. 6, n. 2, p. 206212, Aug. 1992.

KORNING, J.; BALSLEV, H. Growth and mortality of trees in Amazonian tropical rain forest in Ecuador. Journal of Vegetation Science, Knivsta, v. 4, n. 1, p. 77-86, Feb. 1994.

LAURANCE, W. F.; NASCIMENTO, H. E. M.; LAURANCE, S. G.; ANDRADE, A.; RIBEIRO, J.; GIRALDO, J. P.; LOVEJOY, T. E.; CONDIT, R.; CHAVE, J.; HARMS, K. E.; ANGELO, S. Rapid decay of tree-community composition in Amazonian forest fragments. Proceedings of the National Academy of Sciences of the United States of America, Washington, v. 103, n. 50, p. 19010-19014, Oct. 2006.

Cerne, Lavras, v. 18, n. 2, p. 223-230, abr./jun. 2012 
LEUSCHNER, C.; MOSER, G.; BERTSCH, C.; RÖDERSTEIN, M.; HERTEL, D. Large altitudinal increase in tree shroot/shoot ratio in tropical mountain forests of Ecuador. Basic and Applied Ecology, Jena, v. 8, n. 2, p. 219-230, May 2007.

LIEBERMAN, D.; LIEBERMAN, M.; PERALTA, R.; HARTSHORN, G. S. Tropical forest structure and composition on a large-scale altitudinal gradient in Costa Rica. Journal of Ecology, London, v. 84, n. 2, p. 137-152, Apr. 1996.

MICHALSKI, F.; NISHI, I.; PERES, C. A. Disturbancemediated drift in tree functional groups in Amazonian forest fragments. Biotropica, Washington, v. 39, n. 6, p. 691-701, Dec. 2007.

OGDEN, J.; BASHER, L.; MCGLONE, M. Fire, forest regeneration and links with early human habitation: evidence from New Zealand. Annals of Botany, London, v. 81, n. 6, p. 687-696, June 1998.

OLIVEIRA FILHO, A. T.; CARVALHO, W. A. C.; MACHADO, E. L. M.; HIGUCHI, P.; CASTRO, G. C.; SILVA, A. C.; SANTOS, R. M.; BORGES, L. F. R.; CORRÊA, B. S.; ALVES, J. M. Dinâmica da comunidade e populações arbóreas da borda e interior de um remanescente florestal na serra da Mantiqueira, Minas Gerais, em um intervalo de cinco anos: 1999-2004. Revista Brasileira de Botânica, São Paulo, v. 30, n. 1, p. 149-161, Mar. 2007.

OLIVEIRA FILHO, A. T.; FONTES, M. A. L. Patterns of floristic differentiation among Atlantic forests in south-eastern Brazil, and the influence of climate. Biotropica, Washington, v. 31, n. 4, p. 71-88, Apr. 2000.

OLIVEIRA FILHO, A. T.; MELLO, J. M.; SCOLFORO, J. R. S. Effects of past disturbance and edges on tree community structure and dynamics within a fragment of tropical semideciduous forest in south-eastern Brazil over a five-year period: 1987-1992. Plant Ecology, Dordrecht, v. 131, n. 1, p. 45-66, Jan. 1997.

PEREIRA, I. M.; OLIVEIRA FILHO, A. T.; BOTELHO, S. A.; CARVALHO, W. A. C.; FONTES, M. A. L.; SCHIAVINI, I.; SILVA, A. F. Composição florística do compartimento arbóreo de cinco remanescentes florestais do maciço do Itatiaia, Minas Gerais e Rio de Janeiro. Rodriguésia, Rio de Janeiro, v. 57, n. 1, p. 103-126, jan. 2006.

Cerne, Lavras, v. 18, n. 2, p. 223-230, abr./jun. 2012
SHEIL, D. Evaluating turnover in tropical forests. Science, Washington, v. 268, n. 5212, p. 894-895, 1995.

SHEIL, D.; BURSLEM, D. F. R. P. Disturbing hypotheses in tropical forests. Trends in Ecology and Evolution, Amsterdam, v. 18, n. 1, p. 18-26, Jan. 2003.

SHEIL, D.; BURSLEM, D. F. R. P.; ALDER, D. The interpretation and misinterpretation of mortality rate measures. Journal of Ecology, London, v. 83, n. 2, p. 331-333, Apr. 1995.

SHEIL, D.; JENNINGS, S.; SAVILL, P. Long-term permanent plot observations of vegetation dynamics in Bundongo, a Ugandan rain forest. Journal of Tropical Ecology, Cambridge, v. 16, n. 1, p. 765-800, Jan. 2000.

SOETHE, N.; WILCKE, W.; HOMEIER, J.; LEHMANN, J.; ENGELS, C. Plant growth along the altitudinal gradient: role of plant nutritional status, fine root activity and soil properties. Forest Ecology and Management, Amsterdam, v. 151, n. 1/3, p. 139-149, Mar. 2001.

SOKAL, R. R.; ROHLF, F. J. Biometry: the principles and practices of statistics in biological research. New York: Freeman, 1995. 887 p.

SWAINE, M. D.; HALL, J. B.; ALEXANDER, I. J. Tree populations dynamics at Kade, Ghana: 1968-1982. Journal of Tropical Ecology, Cambridge, v. 3, n. 4, p. 331-345, Nov. 1987.

WERNECK, M. S.; FRANCESCHINELLI, E. V.; TAMEIRÃO-NETO, E. Mudanças na florística e estrutura de uma floresta decídua durante um período de quatro anos, 1994-1998, na região do Triângulo Mineiro, MG. Revista Brasileira de Botânica, São Paulo, v. 23, n. 4, p. 399-411, jul./ago. 2000.

WHITE, P. S.; PICKETT, S. T. A. Natural disturbance and patch dynamics: an introduction. In: PICKETT, S. T. A.; WHITE, P. S. (Ed.). The ecology of natural disturbance and patch dvnamics. New York: Academic, 1985. p. 3-13.

WHITMORE, T. C. An introduction to tropical rain forests. Oxford: Clarendon, 1990. 226 p. 